\title{
Crystal structure of bis(4-bromophenylaminium) tetraiodoplumbate(II), $\left(\mathrm{BrC}_{6} \mathrm{H}_{4} \mathrm{NH}_{3}\right)_{2} \mathrm{PbI}_{4}$
}

\author{
Hongxia Dai ${ }^{\mathrm{III}}$, Zhi Liu."., Jiandong Fan', Jianwu Wang ${ }^{\mathrm{II}}$, Xutang Tao' and Minhua Jiang ${ }^{1}$ \\ I Shandong University, State Key Laboratory of Crystal Materials, Jinan, Shandong 250100, P. R. China \\ In Shandong University, School of Chemistry and Chemical Engineering, Jinan, Shandong 250100, P. R. China
}

Received October 31, 2008, accepted and available on-line January 31, 2009; CCDC no. 1267/2495

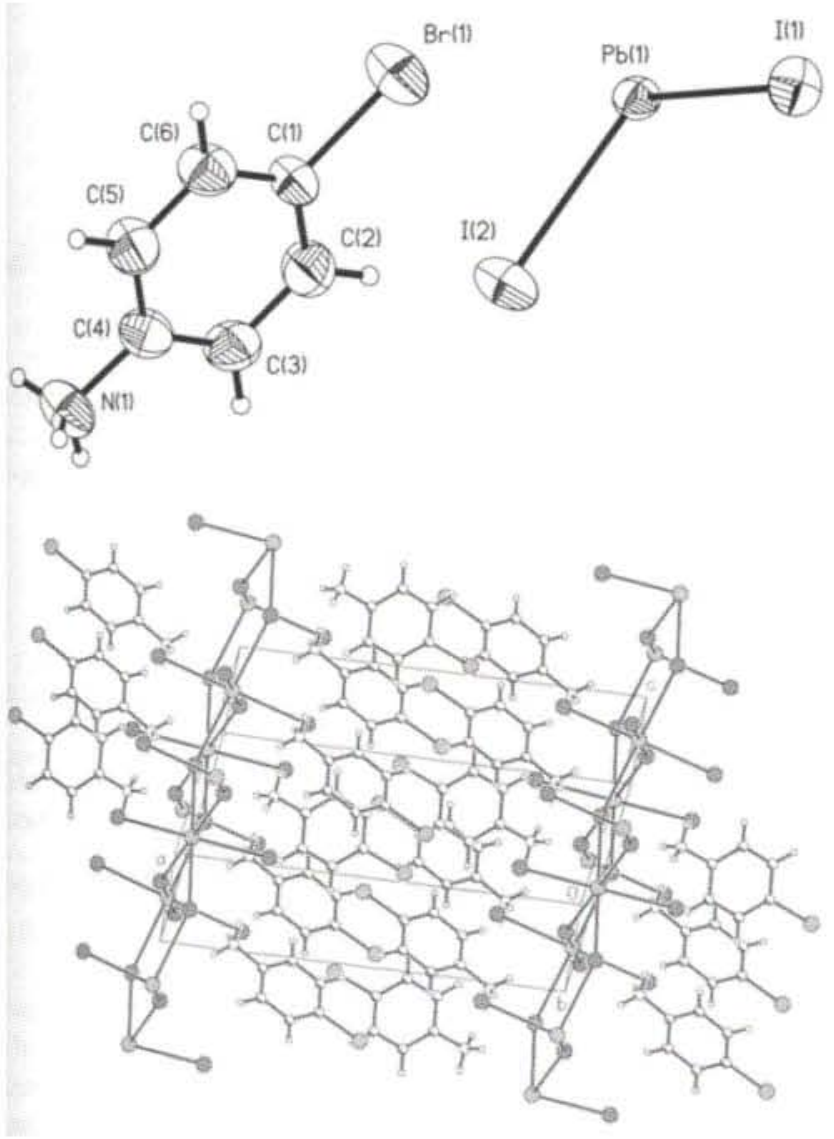

Abstract

$\mathrm{C}_{12} \mathrm{H}_{14} \mathrm{Br}_{2} \mathrm{~L}_{4} \mathrm{~N}_{2} \mathrm{~Pb}$, monoclinic, $P 12_{1} / c 1$ (no. 14), $a=15.5755(2) \AA, b=8.2019(1) \AA, c=9.0917(1) \AA$, $\beta=100.084(1)^{\circ}, V=1143.5 \AA^{3}, Z=2, R_{g r}(F)=0.031$, $w R_{\text {ref }}\left(F^{2}\right)=0.066, T=293 \mathrm{~K}$.

\section{Source of material}

Crystals of the title compound were prepared by slow-cooling method similar to [1,2], with all synthetic processes being operated in dry argon atmosphere or in vacuum. $1.73 \mathrm{~g}(0.01 \mathrm{~mol})$ of $p$ bromoaniline was dissolved in the mixture of concentrated (45.0 $\%)$ aqueous hydroiodic acid and anhydrous ethanol $(v / v=3: 2)$ under following argon at $60^{\circ} \mathrm{C}$. The concentrated $(45.0 \%)$ aqueous $\mathrm{HI}$ solution of $2.30 \mathrm{~g}$ ( $5.0 \mathrm{mmol})$ of $\mathrm{PbI}_{2}$ then dripped from the constant pressure funnel to the above solution gradually. The resulting solution was allowed to sit at $60^{\circ} \mathrm{C}$ for 4 hours and then slowly $\left(5^{\circ} \mathrm{C} / \mathrm{h}\right)$ cooled to room temperature. The precipitate was filtered off. After drying in vacuum, yellow plate-like crystals
$(3.52 \mathrm{~g}, 66.4 \%)$ of high quality were obtained. Elemental analysis - found: $\mathrm{C}, 12.88 \% ; \mathrm{H}, 1.335 \% ; \mathrm{N}, 2.606 \%$; calculated for $\mathrm{C}_{12} \mathrm{H}_{14} \mathrm{Cl}_{2} \mathrm{I}_{4} \mathrm{~N}_{2} \mathrm{~Pb}: \mathrm{C}, 13.59 \% ; \mathrm{H}, 1.33 \% ; \mathrm{N}, 2.64 \%$.

\section{Experimental details}

All $\mathrm{H}$ atoms were positioned geometrically $(d(\mathrm{C}-\mathrm{H})=0.93$ $0.98 \AA)$, and refined as riding with $U_{\text {iso }}(\mathrm{H})=1.2 U_{\text {eq }}$ (carrier) or $1.5 U_{\text {eq }}$ (amino groups).

\section{Discussion}

The title organic-inorganic hybrid $\left(\mathrm{BrC}_{6} \mathrm{H}_{4} \mathrm{NH}_{3}\right)_{2} \mathrm{PbI}_{4}$ is isostructural to $\left(\mathrm{ClC}_{6} \mathrm{H}_{4} \mathrm{NH}_{3}\right)_{2} \mathrm{PbI}_{4}$ (figure, top) [3]. The crystal structure of the title compound contains alternate well-ordered inorganic perovskite layers and organic bimolecular layers along [100]. A corner-sharing distorted $\mathrm{PbI}_{6}$ octahedron connects neighboring ones through four bridging iodine atoms to constitute the former, and two kinds of parallel 4-bromophenylammonium cations form the latter (figure, bottom). Hydrogens of organic cations bond to iodines in the perovskite layers through the ammonium groups and $\mathrm{C}-\mathrm{H}$ groups. The perovskite sheets contain one independent $\mathrm{Pb}$ atom, which has a distorted octahedral iodine coordination with $\mathrm{Pb}-\mathrm{I}$ bond lengths ranging from $3.1690(4) \AA$ to $3.2255(3) \AA$ and $\mathrm{I}-\mathrm{Pb}-\mathrm{I}$ bond angles ranging from $86.361(4)^{\circ}$ to $93.639(4)^{\circ}$, and even to $180.0^{\circ}$. The average $\mathrm{Pb}-\mathrm{I}$ bond length for the $\mathrm{PbI}_{6}$ octahedron is 3.2095(3) $\AA$. These values are similar to those found in $\left(\mathrm{ClC}_{6} \mathrm{H}_{4} \mathrm{NH}_{3}\right)_{2} \mathrm{PbI}_{4}$ and (BAESBT) $\mathrm{PbI}_{4}$ (BAESBT $=5,5^{\prime}$-bis(ammoniumethylsulfanyl)-2,2-bithiophene) [4]. The coordination type involving $\mathrm{PbI}_{6}$ of the title compound differs from those observed in $\left(\mathrm{C}_{12} \mathrm{H}_{14} \mathrm{NH}_{2}\right)_{2} \mathrm{~Pb}_{2} \mathrm{I}_{6}$ [5], which has three coordination types, namely, nearly ideal octahedral symmetry, distorted octahedral coordination and trigonal prismatic coordination. Face-shared $\mathrm{PbI}_{6}$ octahedra and trigonal prisms link with one another to form chains. In $\left(\mathrm{C}_{12} \mathrm{H}_{14} \mathrm{NH}_{2}\right)_{2} \mathrm{~Pb}_{2} \mathrm{I}_{6}, d(\mathrm{~Pb}-\mathrm{I})$ are between 3.173(1) $\AA$ to $3.346(1) \AA$ and $\mathrm{I}-\mathrm{Pb}-\mathrm{I}$ bond angles range from $82.5^{\circ}$ to $90.0^{\circ}$ and $166.65^{\circ}$ to $180.0^{\circ}$. The organic layers comprise two kinds of parallel 4-bromo-phenylammonium cations with the dihedral angel of $37.75(2)^{\circ}$, whose planes are defined by $\mathrm{Br} 1 / \mathrm{C} 1 / \mathrm{C} 2 / \mathrm{C} 3 / \mathrm{C} 4 / \mathrm{C} 5 / \mathrm{C} 6 / \mathrm{N} 1$. Besides electrostatic interaction, classic and non-classic hydrogen bonds between organic bimolecular layers and inorganic layers exist to maintain stability of the crystal structure.

\footnotetext{
* Correspondence author (e-mail: lz@sdu.edu.cn)
} 
Table 1. Data collection and handling.

\begin{tabular}{ll}
\hline Crystal: & yellow plate, size $0.03 \times 0.07 \times 0.35 \mathrm{~mm}$ \\
Wavelength: & Mo $K_{\alpha}$ radiation $(0.71073 \AA)$ \\
$\mu:$ & $162.67 \mathrm{~cm}^{-1}$ \\
Diffractometer, scan mode: & Bruker APEX2 CCD, $\varphi / \omega$ \\
$2 \theta_{\text {max }}$ & $54.98^{\circ}$ \\
$N(h k l)_{\text {measured }} N(h k l)_{\text {unique }}:$ & 7918,2613 \\
Criterion for $I_{\text {obs, }} N(h k l)_{\text {gi }}:$ & $I_{\text {obs }}>2 \sigma\left(I_{\text {obs }}\right), 2329$ \\
$N(\text { param })_{\text {refined: }}$ & 99 \\
Programs: & SHELXL-97 [6], SHELXTL [7], \\
& WinGX [8]
\end{tabular}

Table 2. Atomic coordinates and displacement parameters (in $\AA^{2}$ ).

\begin{tabular}{llllll}
\hline Atom & Site & $x$ & $y$ & $z$ & $U_{\text {iso }}$ \\
\hline $\mathrm{H}(2)$ & $4 e$ & 0.5450 & 0.3339 & 0.5011 & 0.068 \\
$\mathrm{H}(3)$ & $4 e$ & 0.6857 & 0.3780 & 0.6296 & 0.063 \\
$\mathrm{H}(5)$ & $4 e$ & 0.7573 & 0.5293 & 0.2497 & 0.064 \\
$\mathrm{H}(6)$ & $4 e$ & 0.6169 & 0.4827 & 0.1223 & 0.062 \\
$\mathrm{H}(1 \mathrm{~A})$ & $4 e$ & 0.8596 & 0.4378 & 0.4977 & 0.088 \\
$\mathrm{H}(1 \mathrm{~B})$ & $4 e$ & 0.8233 & 0.4841 & 0.6283 & 0.088 \\
$\mathrm{H}(1 \mathrm{C})$ & $4 e$ & 0.8317 & 0.6047 & 0.5147 & 0.088
\end{tabular}

Table 3. Atomic coordinates and displacement parameters (in $\AA^{2}$ ).

\begin{tabular}{|c|c|c|c|c|c|c|c|c|c|c|}
\hline Atom & Site & $x$ & $y$ & $z$ & $U_{11}$ & $U_{22}$ & $U_{33}$ & $U_{12}$ & $U_{13}$ & $U_{23}$ \\
\hline $\operatorname{Br}(1)$ & $4 e$ & $0.45139(5)$ & $0.37977(8)$ & $0.20091(7)$ & $0.0473(5)$ & $0.0874(4)$ & $0.0603(3)$ & $-0.0058(3)$ & $-0.0008(3)$ & $-0.0099(3)$ \\
\hline$C(1)$ & $4 e$ & $0.5686(4)$ & $0.4067(6)$ & $0.3003(5)$ & $0.033(3)$ & $0.052(3)$ & $0.043(2)$ & $-0.003(2)$ & $0.003(2)$ & $-0.003(2)$ \\
\hline$C(2)$ & $4 e$ & $0.5880(5)$ & $0.3737(7)$ & $0.4512(6)$ & $0.052(4)$ & $0.071(4)$ & $0.047(2)$ & $-0.010(3)$ & $0.008(3)$ & $0.010(2)$ \\
\hline$C(3)$ & $4 e$ & $0.6715(4)$ & $0.4001(6)$ & $0.5279(5)$ & $0.055(4)$ & $0.066(4)$ & $0.036(2)$ & $-0.001(3)$ & $0.007(3)$ & $0.009(2)$ \\
\hline$C(4)$ & $4 e$ & $0.7330(4)$ & $0.4593(6)$ & $0.4517(5)$ & $0.046(4)$ & $0.053(3)$ & $0.039(2)$ & $0.004(3)$ & $0.003(3)$ & $-0.005(2)$ \\
\hline$C(5)$ & $4 e$ & $0.7143(5)$ & $0.4905(6)$ & $0.2999(6)$ & $0.053(5)$ & $0.072(4)$ & $0.035(2)$ & $-0.008(3)$ & $0.010(3)$ & $-0.000(2)$ \\
\hline I(1) & $4 e$ & $-0.03871(3)$ & $0.17976(4)$ & $0.79710(3)$ & $0.0604(3)$ & $0.0442(2)$ & $0.0388(2)$ & $-0.0053(2)$ & $0.0128(2)$ & $-0.0149(1)$ \\
\hline $\mathrm{I}(2)$ & $4 e$ & $0.20191(3)$ & $0.42119(5)$ & $1.08206(3)$ & $0.0369(3)$ & $0.0830(3)$ & $0.0404(2)$ & $0.0067(2)$ & $0.0024(2)$ & $-0.0054(1)$ \\
\hline$N(1)$ & $4 e$ & $0.8205(4)$ & $0.5005(6)$ & $0.5309(5)$ & $0.042(4)$ & $0.090(4)$ & $0.043(2)$ & $-0.002(2)$ & $0.004(2)$ & $-0.010(2)$ \\
\hline $\mathrm{Pb}(1)$ & $2 c$ & 0 & $1 / 2$ & 0 & $0.0407(2)$ & $0.0381(2)$ & $0.0273(1)$ & $-0.0009(1)$ & $0.0024(1)$ & $-0.00019(7)$ \\
\hline
\end{tabular}

Acknowledgment. This work was supported by the National Natural Science Foundation of China (grant nos. 50402018, 57021002).

\section{References}

1. Mitzi, D. B.: A Layered Solution Crystal Growth Technique and the Crystal Structure of $\left(\mathrm{C}_{6} \mathrm{H}_{5} \mathrm{C}_{2} \mathrm{H}_{4} \mathrm{NH}_{3}\right)_{2} \mathrm{PbCl}_{4}$. J. Solid State Chem. 145 (1999) 694-704.

2. Liu, Z.; Yu, W.-T.; Tao, X.-T.; Jiang, M.-H.: Crystal structure of bis(4bromoanilinium) tetrachloroplumbate(II), (4-Br- $\left.\mathrm{C}_{6} \mathrm{H}_{4} \mathrm{NH}_{3}\right)_{2} \mathrm{PbCl}_{4} . \mathrm{Z}$. Kristallogr. NCS 219 (2004) 303-304.

3. Liu, Z.; Yu, W.-T.; Tao, X.-T.; Jiang, M.-H.: Crystal structure of bis(4 chlorophenylaminium) tetraiodoplumbate(II), (4-Cl- $\left.\mathrm{C}_{6} \mathrm{H}_{4} \mathrm{NH}_{3}\right)_{2} \mathrm{PbL}_{4} . \mathrm{Z}$. Kristallogr. NCS 219 (2004) 457-458.

4. Zhu, X. H.; Mercier, N.; Frere, P.; Blanchard, P.; Roncali, J.; Allain, M.; Pasquier, C.; Riou, A.: Effect of Mono- versus Di-ammonium Cation of 2,2'-Bithiophene Derivatives on the Structure of Organic-Inorganic $\mathrm{Hy}$ brid Materials Based on Iodo Metallates. Inorg. Chem. 42 (2003) 5330 5339.
5. Tang, Z.; Guloy, A. M.: Methylviologen Lead(II) Iodide: Novel [ $\mathrm{PbI}_{3}{ }^{-}$] Chains with Mixed Octahedral and Trigonal Prismatic Coordination. J. Am. Chem. Soc. 121 (1999) 452-453.

6. Sheldrick, G. M.: SHELXL-97. Program for the Refinement of Crystal Structures. University of Göttingen, Germany 1997.

7. Sheldrick, G. M.: SHELXTL. Structure Determination Software Suite. Version 5.10. Bruker AXS, Madison, Wisconsin, USA 1997.

8. Farrugia, L. J.: WinGX suite for small-molecule single-crystal crystallogrphy. J. Appl. Crystallogr. 32 (1999) 837-838. 